ORIGINAL PAPER

\title{
Clinical and prognostic Significance of KrüPPel-Like TRANSCRIPTION FACTOR 6 EXPRESSION IN 67 PATIENTS WITH CUTANEOUS MALIGNANT MELANOMA IN CHINA
}

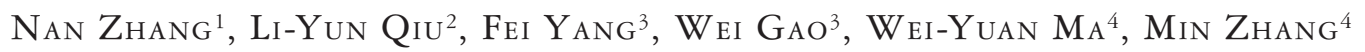

${ }^{1}$ Department of Oncology, Jinan Central Hospital, Cheeloo College of Medicine, Shandong University, Jinan, Shandong, PR China

${ }^{2}$ Department of Pharmacy, J Jinan Central Hospital, Cheeloo College of Medicine, Shandong University, Jinan, Shandong, PR China

${ }^{3}$ Department of Pathology, Jinan Central Hospital, Cheeloo College of Medicine, Shandong University, Jinan, Shandong, PR China

${ }^{4}$ Department of Dermatology, Qilu Hospital, Shandong University, Jinan, Shandong Province, PR China

\begin{abstract}
Krüppel-like transcription factor $6(\mathrm{KLF} 6)$ is a ubiquitous tumor suppressor gene involved in regulating cell growth, proliferation, differentiation and angiogenesis. The objective of the study was to investigate the clinical and prognostic significance of KLF6 expression in cutaneous malignant melanoma (CMM) patients. A total of CMM 67 patients were enrolled in this study. The specimens were evaluated by immunohistochemistry to detect KLF6. The positive KLF6 expression in CMM tissues was significantly lower than in normal skin tissues $(\mathrm{p}<0.01)$. The presence of KLF6 in CMM was correlated with ulceration ( $<<0.01)$, lymph node metastasis $(\mathrm{p}<0.01)$ and clinical stage $(\mathrm{p}<0.01)$. The overall 5 -year survival rate of the 67 patients was $13.4 \%$. The 5 -year survival rate of patients with negative KLF6 expression was correlated with KLF6 expression ( $\mathrm{p}<0.01)$, ulceration ( $\mathrm{p}<0.01)$, lymph node metastasis $(\mathrm{p}<0.01)$, clinical stage $(\mathrm{p}<0.01)$ and operation type $(\mathrm{p}<0.01)$. Ulceration and clinical staging were independent relevant factors. In conclusion, the presence of KLF6 in CMM was correlated with ulceration, lymph node metastasis, clinical stage and poor prognosis.
\end{abstract}

Key words: KLF6, cutaneous malignant melanoma, prognosis, immunohistochemistry.

\section{Introduction}

Cutaneous malignant melanoma (CMM) is a common malignant tumor in western countries and is known for its rapid progression, metastasis, and poor prognosis [1]. It is relatively rare in Asians. In recent years, CMM has been one of the most rapidly increasing cancers in China [2]. The TNM staging system is widely used to guide therapeutic decisions and prognosis in patients with CMM in the current practice.
However, it often could not supply the precise outcome to the individual patient. Recently some molecular biomarkers combined with the TNM staging system might be valuable to accurately distinguish CMM patients' prognosis.

The Krüppel-like transcription factor 6 (KLF6) is a member of the Krüppel-like factor family. It belong to a group of zinc finger transcription factors $[3,4]$. Recent studies show that KLF6 is a ubiquitous tumor suppressor gene involved in regulating cell growth, 

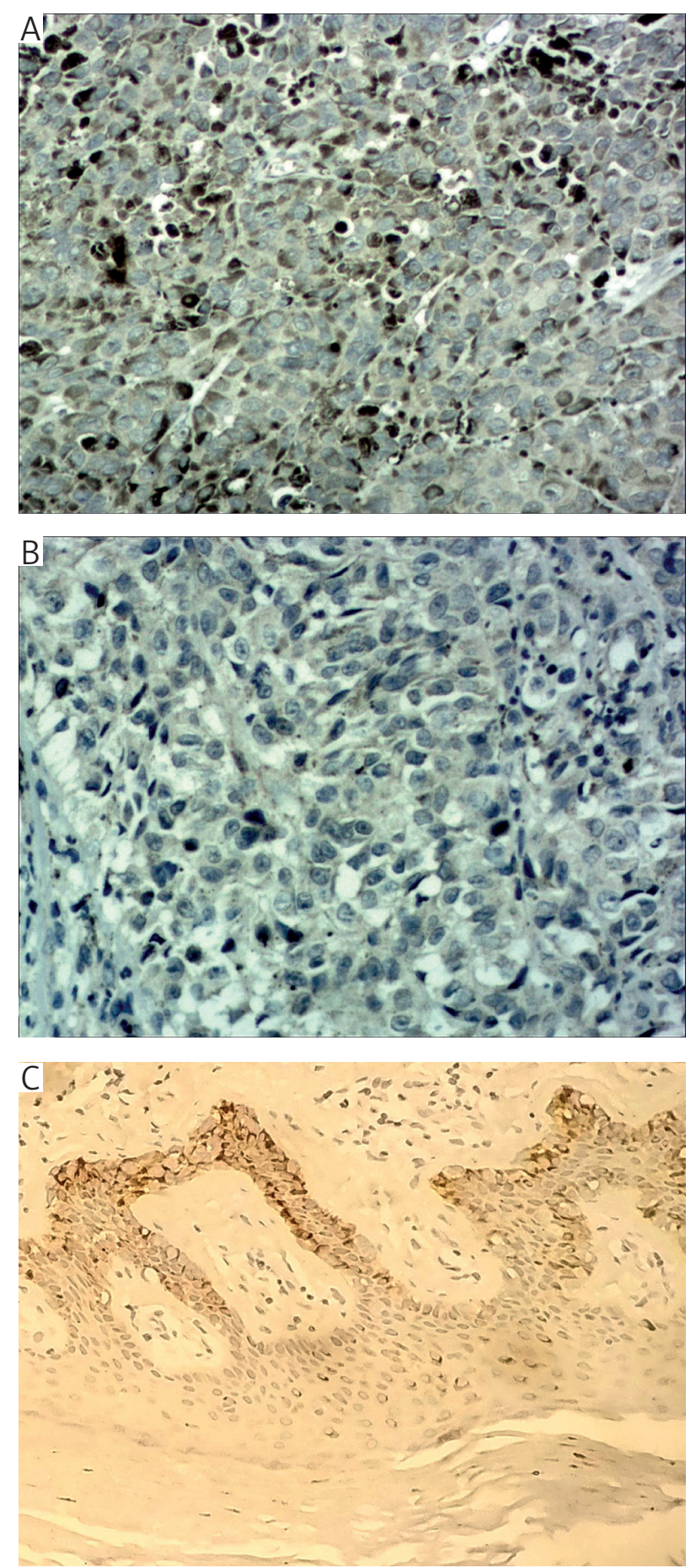

Fig. 1. A) Immunohistochemical staining of human CMM tissue sections demonstrating KLF6 protein. The KLF6 staining was mainly located in the cytoplasm, and photomicrographs showed human CMM specimen (foot) with high KLF6 expression tumor cells $(\geq 7)$. Original magnification $\times 200$; B) Photomicrographs showing CMM specimen (foot) with low KLF6 expression tumor cells $(\leq 6)$. Original magnification $\times 200$; C) Photomicrographs showing the corresponding normal tissue specimen (neck) with high KLF6 expression normal cells $(\geq 7)$. Original magnification $\times 200$ proliferation, differentiation and angiogenesis. It is closely associated with the occurrence of prostate cancer, liver cancer, lung cancer, etc. $[5,6,7]$. It is also reported that KLF6 is an important prognostic factor in some cancers [8]. There has been limited research confirming the correlation between KLF6 expression and clinic pathological value in CMM. Thus, we designed the present study to investigate the clinical and prognostic significance of KLF6 expression in CMM patients by both univariate and multivariate analysis. Also we detected KLF6 expression by immunohistochemistry.

\section{Material and methods}

\section{Patients}

A total of 67 patients were enrolled in this study at the Department of Dermatology, Department of Oncology, Jinan Central Hospital Affiliated to Shandong University and the Department of Dermatology, Qilu Hospital between January 2001 and December 2011. The inclusion criteria were as follows: (1) the pathology confirmed CMM by surgery or biopsy; (2) TNM staging according to the American Joint Committee on Cancer (AJCC) staging system (6th edition); (3) patients accepted no preoperative radiotherapy or chemotherapy; (4) the cases have been well preserved. The clinicopathological characteristics of the 67 patients are listed in Table I. This study was approved by Shandong University Ethics Committee.

\section{Immunohistochemistry}

All the CMM specimens were obtained from the 67 patients. The 10 control specimens were obtained from areas of normal skin tissue, taken from the patients who underwent surgery (healthy tissue sites were $3 \mathrm{~cm}$ away from the CMM tissue). The tissue specimens were fixed in $10 \%$ neutral buffered formalin and processed routinely. Hematoxylin and eosin (HE)-stained slides as well as immunohistochemical reactions were performed on paraffin-embedded and formalin-fixed tissue using rabbit antihuman KLF6 monoclonal antibodies (Abcam, Cambridge, UK; $1: 100$ dilution) and visualized by the Envision System (Dako). The presence of KLF6 was determined by light microscopy (BX51, Olympus Optical, Tokyo, Japan). KLF6 was mainly located in the tumor cell cytoplasm. KLF6 protein expression is indicated by yellow-to-brown color and was scored by the semiquantitative immunoreactivity scoring system (IRS), as follows: staining intensity $(0=$ no staining, $1=$ weak staining, $2=$ moderate staining, $3=$ strong staining) and the percentage of cells stained $(0=$ no cells, $1=1-10 \%$ of cells, $2=11-50 \%$ 
Table I. Correlation between KLF6 expression and clinical features of the CMM patients

\begin{tabular}{|c|c|c|c|c|}
\hline \multirow[t]{3}{*}{$\begin{array}{l}\text { CLINICAL } \\
\text { CHARACTERISTICS }\end{array}$} & \multirow[t]{2}{*}{ Patient } & \multicolumn{2}{|c|}{$\begin{array}{c}\text { KLF6 } \\
\text { EXPRESSION }\end{array}$} & \multirow[t]{2}{*}{$\begin{array}{c}\mathbf{P} \\
\text { VALUE }^{A}\end{array}$} \\
\hline & & Low & HigH & \\
\hline & 67 & 50 & 17 & \\
\hline Gender & & & & $0.159^{*}$ \\
\hline Male & 36 & 24 & 12 & \\
\hline Female & 31 & 26 & 5 & \\
\hline Age, years & & & & $1.000 *$ \\
\hline$<50$ & 33 & 25 & 8 & \\
\hline$\geq 50$ & 34 & 25 & 9 & \\
\hline Locality & & & & $>0.05$ \\
\hline Head and neck & 6 & 4 & 2 & \\
\hline Trunk & 10 & 8 & 2 & \\
\hline Extremities & 51 & 38 & 13 & \\
\hline Ulceration & & & & $0.001 *$ \\
\hline Yes & 26 & 13 & 13 & \\
\hline No & 41 & 37 & 4 & \\
\hline Histological type & & & & $>0.05$ \\
\hline $\mathrm{NM}$ & 12 & 11 & 1 & \\
\hline SSM & 49 & 36 & 13 & \\
\hline ALM & 6 & 3 & 3 & \\
\hline $\mathrm{pN}$ & & & & $0.004 *$ \\
\hline- & 26 & 13 & 13 & \\
\hline+ & 41 & 37 & 4 & \\
\hline Clinical stage & & & & $<0.01$ \\
\hline I & 4 & 0 & & \\
\hline II & 16 & 9 & & \\
\hline III & 33 & 28 & & \\
\hline IV & 14 & 13 & & \\
\hline
\end{tabular}

of cells, $3=51-80 \%$ of cells, $4=81-100 \%$ of cells) were evaluated and the respective scores were multiplied, resulting in an IRS range from 0 to 12 . For statistical analysis, cases were grouped as either low expression (IRS 0-6) or high expression (IRS 7-12), according to previous reports [9].

\section{Statistical methods}

Fisher's exact probability test or the $\chi^{2}$ test was used to calculate the correlation between KLF6 expression and clinicopathological factors. Univariate analysis was carried out by the use of the $\mathrm{Ka}$ plan-Meier survival curves. Multivariate analysis was analyzed using the Cox proportional hazard model. When the $\mathrm{p}$ value was less than 0.05 , we considered

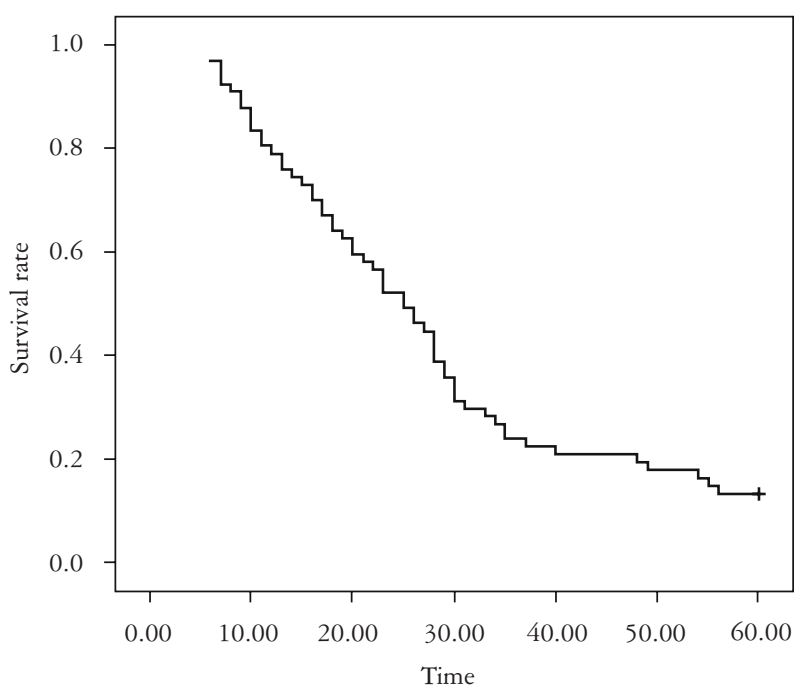

Fig. 2. Kaplan-Meier analysis of overall survival

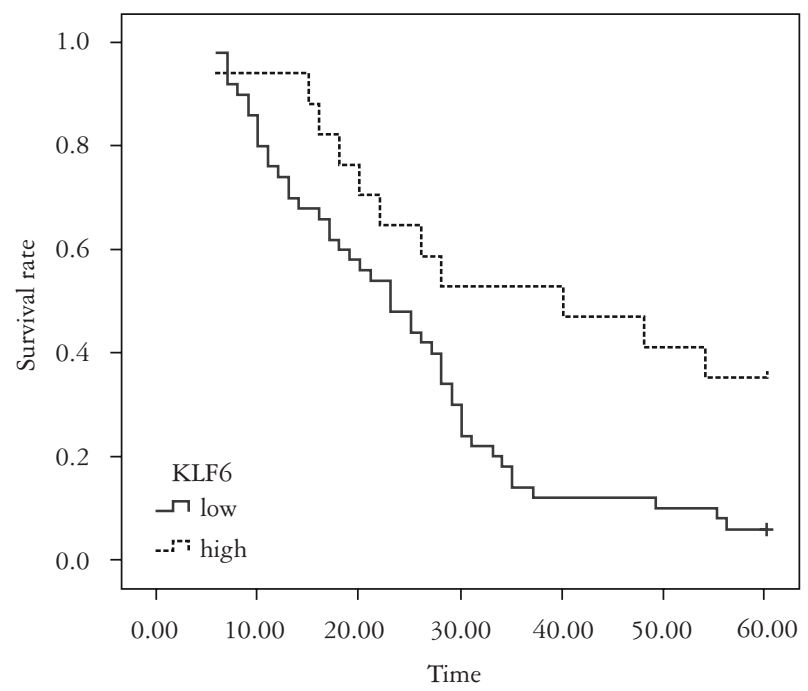

Fig. 3. Kaplan-Meier analysis of overall survival rate in patients with KLF6 expression

the differences significant. The obtained data were analyzed using the statistical software package SPSS (SPSS 13.0, SPSS Inc., Chicago, IL, USA).

\section{Results}

The protein KLF6 was found in normal tissues and malignant melanoma lesions: positive staining of a yellow-to-brown color was mainly observed in the epithelial prickle and basal layers in normal tissues; in malignant melanoma lesions, yellow staining was observed in the cuticular layer. CMM tissue from 17 patients was positive for KLF6, giving a positive rate of $25.4 \%$, which was significantly lower compared with control tissues $(\mathrm{p}<0.01)$ (Fig. 1). The presence of KLF6 in CMM was correlated with ulceration $(\mathrm{p}<0.01)$, lymph node metastasis $(\mathrm{p}<0.01)$ and clinical stage $(\mathrm{p}<0.01)$. There was 
Table II. Results of univariate survival analysis of the CMM patients

\begin{tabular}{|c|c|c|c|c|}
\hline \multirow[t]{3}{*}{ CLINICAL CHARACTERISTICS } & \multirow{2}{*}{$\begin{array}{c}\text { Patients } \\
67\end{array}$} & \multicolumn{2}{|c|}{ 5-YEAR SURVIVAL (\%) } & \multirow[t]{3}{*}{$\mathbf{P}$ value } \\
\hline & & Patients & RATE $(\%)$ & \\
\hline & & 9 & 13.4 & \\
\hline Gender & & & & 0.080 \\
\hline Male & 36 & 8 & 22.2 & \\
\hline Female & 30 & 1 & 3.3 & \\
\hline Age (years) & & & & 0.316 \\
\hline$<50$ & 33 & 4 & 12.1 & \\
\hline$\geq 50$ & 34 & 5 & 14.7 & \\
\hline Locality & & & & 0.361 \\
\hline head and neck & 6 & 1 & 16.7 & \\
\hline trunk & 10 & 1 & 10.0 & \\
\hline extremities & 51 & 7 & 13.7 & \\
\hline Ulceration & & & & 0.000 \\
\hline yes & 41 & 0 & 0 & \\
\hline no & 26 & 9 & 34.6 & \\
\hline Histological type & & & & 0.171 \\
\hline NM & 12 & 1 & 8.3 & \\
\hline SSM & 6 & 2 & 33.3 & \\
\hline ALM & 49 & 6 & 12.2 & \\
\hline $\mathrm{pN}$ & & & & 0.000 \\
\hline- & 26 & 9 & 34.6 & \\
\hline+ & 41 & 0 & 0 & \\
\hline Clinical stage & & & & $<0.01$ \\
\hline I & 4 & 3 & 75.0 & \\
\hline II & 16 & 6 & 37.5 & \\
\hline III & 33 & 0 & 0.0 & \\
\hline IV & 14 & 0 & 0.0 & \\
\hline Operative type & & & & 0.000 \\
\hline Surgery & 46 & 9 & 19.6 & \\
\hline Biopsy & 21 & 0 & 0.0 & \\
\hline Chemotherapy & & & & 0.637 \\
\hline Yes & 23 & 5 & 21.7 & \\
\hline No & 44 & 4 & 9.1 & \\
\hline Radiotherapy & & & & 0.707 \\
\hline Yes & 8 & 2 & 25.0 & \\
\hline No & 59 & 7 & 11.9 & \\
\hline Immunotherapy & & & & 0.809 \\
\hline Yes & 53 & 7 & 13.2 & \\
\hline No & 14 & 2 & 14.3 & \\
\hline KLF6 expression & & & & 0.008 \\
\hline Low & 50 & 3 & 6.0 & \\
\hline High & 17 & 6 & 35.3 & \\
\hline
\end{tabular}

$P$ values were obtained with the log-rank test 
Table III. Results of multivariate Cox regression analysis of survival

\begin{tabular}{|c|c|c|c|c|c|c|}
\hline & B & SE & WALD & $\mathbf{P}$ & HR & $95 \%$ CI FOR HR \\
\hline Gender & -0.003 & 0.317 & 0.000 & 0.992 & 0.997 & $0.535-1.856$ \\
\hline Age & -0.179 & 0.151 & 1.408 & 0.235 & 0.836 & $0.623-1.124$ \\
\hline Locality & 0.523 & 0.275 & 3.612 & 0.057 & 1.687 & $0.984-2.894$ \\
\hline Ulceration & 0.983 & 0.464 & 4.495 & 0.034 & 2.672 & $1.077-6.630$ \\
\hline Histological type & 0.136 & 0.195 & 0.487 & 0.485 & 1.146 & $0.781-1.681$ \\
\hline $\mathrm{pN}$ & 0.036 & 0.486 & 0.005 & 0.941 & 1.037 & $0.400-2.689$ \\
\hline Clinical stage & 1.411 & 0.375 & 14.173 & 0.000 & 4.102 & $1.967-8.553$ \\
\hline Operative type & -0.202 & 0.346 & 0.339 & 0.561 & 0.817 & $0.415-1.612$ \\
\hline Chemotherapy & -0.506 & 0.388 & 1.695 & 0.193 & 0.603 & $0.282-1.291$ \\
\hline Radiotherapy & -0.676 & 0.481 & 1.972 & 0.160 & 0.509 & $0.198-1.306$ \\
\hline Immunotherapy & 0.095 & 0.383 & 0.061 & 0.805 & 1.099 & $0.518-2.330$ \\
\hline KLF6 expression & 0.036 & 0.486 & 0.005 & 0.941 & 1.037 & $0.400-2.689$ \\
\hline
\end{tabular}

$B$ - regression coefficient; SE - standard error; Wald - Wald value; $H R$ - hazard ratio; $C I$ - confidence interval

no significant correlation between KLF6 and other clinicopathological features (Table I).

Among the CMM patients, 46 patients had undergone surgery, 23 patients had adjuvant chemotherapy, 8 patients had adjuvant radiotherapy and 53 patients had immunotherapy. The overall 5-year survival rate of the 67 patients was $13.4 \%$ (Fig. 2). In univariate analysis by the log-rank test, the 5 -year survival rate of patients with negative KLF6 expression was correlated with KLF6 expression ( $\mathrm{p}<0.01)$, ulceration $(\mathrm{p}<0.01)$, lymph node metastasis $(\mathrm{p}<0.01)$, clinical stage $(\mathrm{p}<0.01)$ and operation type $(\mathrm{p}<0.01 ;$ Fig. 3$)$. There was no significant difference among patients according to gender, age, tumor locality, histological type, chemotherapy, radiotherapy or immunotherapy (Table II). The results of Cox regression multivariate analysis showed that ulceration and clinical staging were independent relevant factors (Table III).

\section{Discussion}

The KLF6 gene is regarded as a tumor suppressor on the basis of its inactivation by somatic mutations and loss of heterozygosity in prostate malignant tumor $[10,11]$. Recently, some reports showed that the KLFG gene could also be inactivated in many cancers $[12,13]$. The studies reported that the growth suppressive properties of KLF6 were associated with some cancer pathways, including up-regulating p21 and E-cadherin, down-regulating cyclin D1and CDK4, etc. [14, 15]. However, there are few reports in the literature investigating KLFC mutations in CMM. Cai reported that only $27.5 \%$ of patients had positive expression of KLF6 in CMM tissues [16]. To further clarify the role of KLF6 in CMM, we examined expression of KLF6 in 67 CMM samples by immunohistochemistry. In the study, pos- itive expression of KLF6 in CMM was only found in 17 cases $(25.4 \%)$ and was remarkably lower than expression in normal skin tissue. Statistically, decreased expression of KLF6 was significantly associated with ulceration, lymph node metastasis and clinical stage in CMM. Our results suggested that KLF6 might be involved in tumor development and progression in CMM. However, few studies have reported the clinical and prognostic significance of KLF6 in cancer patients. In Cai's study, the univariate survival analysis showed that the 3-year survival rate in CMM patients with low levels of KLF6 protein was significantly lower than that in patients with high levels of KLF6 $(p<0.05)$ [16]. In our study the overall 5-year survival rate of the $67 \mathrm{CMM}$ patients was $13.4 \%$, and the 5-year survival rate of CMM patients with reduced KLF6 expression was significantly lower than that of patients with positive KLF6 expression, although it was not an independent relevant factor for prognosis.

It has been reported that some clinical characteristics of CMM patients were significantly different among different ethnic groups [17]. Our study showed that $61.2 \%$ of the patients had ulcers at the time of the initial diagnosis. Compared with other races, the primary tumor site is more likely to be an ulcer in the initial diagnosis of Asians [18]. Ulceration has previously been shown in a multivariate analysis to be an independent prognostic indicator of disease-free survival in melanoma patients [19]; but in a study by van Akkoi et al., it did not remain an independent prognostic factor for overall survival after the multivariate analysis [20]. In our study, the 5-year survival rate of patients with ulceration was significantly lower than that of patients without ulceration. Cox regression multivariate analysis 
showed that ulceration was also one of the independent relevant factors for prognosis.

Stage has been repeatedly confirmed to be one of the most important prognostic indicators for melanoma $[21,22]$. However, most evidence presented in the literature originated in endemic regions, particularly western countries, and the most commonly diagnosed subtype of malignant melanoma is superficial spreading melanoma (SSM). Due to the high prevalence of the disease, knowledge on diagnosis and screening is readily available and diagnoses are usually made in relatively earlier stages. Cascinelli reported that the 5-year survival rate of the patients was $80.8 \%$ in the white race in western countries [23]. Our study showed only $29.8 \%$ patients with stage I-II, and the 5-year survival rate in patients was significantly associated with lymph node metastasis and clinical staging. Moreover; clinical staging was one of the independent relevant factors for prognosis in multivariate analysis.

Surgery is standard treatment for localized melanoma. There is no standard therapy for advanced-stage melanoma. In China the indications for treatment not only depend on the Chinese Guidelines for the Diagnosis and Treatment of Melanoma but also on patients' willingness and economic status. In our study, $68.7 \%$ of the patients underwent surgery. The 5-year survival rate of patients who had undergone surgery was significantly higher than that of the other patients. However, Cox multivariate analysis did not show it to be an independent relevant factor.

However, the present study still had several limitations. First, in China the indications for treatment depend not only on doctors' preferences but also on patients' willingness and economic status. These factors might have influenced the relatively poor survival result observed. Secondly, the sample was relatively small.

In conclusion, the presence of KLF6 in CMM was correlated with ulceration, lymph node metastasis and clinical stage. The 5-year survival rate in CMM patients was significantly associated with KLF6 expression, ulceration, lymph node metastasis, clinical stage and clinical operation. Ulceration and clinical staging were independent relevant factors for poor prognosis.

The authors declare no conflict of interest.

This work was supported by the 19th batch of science and technology innovation development plan of Jinan in 2020 (Clinical medicine science and technology innovation plan, grant no. 202019031), and the second group of science and technology projects of Jinan Health Committee (grant no. 2020-4-9).

\section{References}

1. Kadakia S, Chan D, Mourad M, Ducic Y. The Prognostic Value of Age, Sex, and Subsite in Cutaneous Head and Neck Melanoma: A Clinical Review of Recent Literature. Iran J Cancer Prev 2016; 9: e5079.

2. Hao M, Zhao G, Du X, Yang Y, Yang J. Clinical characteristics and prognostic indicators for metastatic melanoma: data from 446 patients in north China. Tumour Biol 2016; 37: 1033910348.

3. Lu XJ, Shi Y, Chen JL, Ma S. Krüppel-like factors in hepatocellular carcinoma. Tumour Biol 2015; 36: 533-541.

4. Sangodkar J, Shi J, DiFeo A, et al. Functional role of the KLF6 tumour suppressor gene in gastric cancer. Eur J Cancer 2009; 45: 666-676.

5. Liu X, Gomez-Pinillos A, Loder C, et al. KLF6 loss of function in human prostate cancer progression is implicated in resistance to androgen deprivation. Am J Pathol 2012; 181: 10071016.

6. Vetter D, Cohen-Naftaly M, Villanueva A, et al. Enhanced hepatocarcinogenesis in mouse models and human hepatocellular carcinoma by coordinate KLF6 depletion and increased messenger RNA splicing. Hepatology 2012; 56: 1361-1370.

7. Tahara E, Kadara H, Lacroix L, et al. Activation of protein kinase $\mathrm{C}$ by phorbol 12-myristate 13-acetate suppresses the growth of lung cancer cells through KLF6 induction. Cancer Biol Ther 2009; 8: 801-807.

8. Khan MS, Bawany FI, Naeem M. Oncogenic splice variant of KLF6 as a prognostic marker in cancers. J Pak Med Assoc 2013; 63: 1215 .

9. Zhang Q, Tan XP, Yuan YS, et al. Decreased expression of KLF6 and its significance in gastric carcinoma. Med Oncol 2010; 27: 1295-1302.

10. Chen C, Hyytinen ER, Sun X, et al. Deletion, mutation, and loss of expression of KLF6 in human prostate cancer. Am J Pathol 2003; 162: 1349-1354.

11. Narla G, Heath KE, Reeves HL, et al. KLF6, a candidate tumor suppressor gene mutated in prostate cancer. Science 2001 294: 2563-2566.

12. DiFeo A, Narla G, Hirshfeld J, et al. Roles of KLF6 and KLF6SV1 in ovarian cancer progression and intraperitoneal dissemination. Clin Cancer Res 2006; 12: 3730-3739.

13. Ghaleb AM, Yang VW. The Pathobiology of Krüppel-like Factors in Colorectal Cancer.Curr Colorectal Cancer Rep 2008; 4 : 59-64.

14. DiFeo A, Martignetti JA, Narla G. The role of KLF6 and its splice variants in cancer therapy. Drug Resist Updat 2009; 12: $1-7$

15. DiFeo A, Narla G, Camacho-Vanegas O, et al. E-cadherin is a novel transcriptional target of the KLF6 tumor suppressor. Oncogene 2006; 25: 6026-6031.

16. Cai D, Zhao J, Sun Q. Kruppel-like factor 6 in the progression and prognosis of malignant melanoma. J Int Med Res 2014; 42: $184-190$.

17. Wu CE, Hsieh CH, Chang CJ, et al. Prognostic factors for Taiwanese patients with cutaneous melanoma undergoing sentinel lymph node biopsy. J Formos Med Assoc 2015; 114 : 415-421.

18. Eggermont AM, Spatz A, Lazar V, Robert C. Is ulceration in cutaneous melanoma just a prognostic and predictive factor or is ulcerated melanoma a distinct biologic entity? Curr Opin Oncol 2012; 24: 137-140.

19. Yee VS, Thompson JF, McKinnon JG, et al. Outcome in 846 cutaneous melanoma patients from a single center after a negative sentinel node biopsy. Ann Surg Oncol 2005; 12: 429-439.

20. Van Akkooi AC, de Wilt JH, Verhoef C, et al. Clinical relevance of melanoma micrometastases $(<0.1 \mathrm{~mm})$ in sentinel nodes: are these nodes to be considered negative? Ann Oncol 2006; $17:$ 1578-1585. 
21. Cormier JN, Xing Y, Ding M, et al. Ethnic differences among patients with cutaneous melanoma. Arch Intern Med 2006; 166: 1907-1914.

22. MacKie RM, Bray C, Vestey J, et al. Melanoma incidence and mortality in Scotland 1979-2003. Br J Cancer 2007; 96 : 1772-1777.

23. Cascinelli N. Margin of resection in the management of primary melanoma. Semin Surg Oncol 1998; 14: 272-275.

\section{Address for correspondence}

\section{Min Zhang}

Department of Dermatology

Jinan Central Hospital

Cheeloo College of Medicine

Shandong University

Jinan, Shandong 250013, PR China

e-mail: zm1447@126.com 